\title{
Contrasting treatment-specific survival using double-robust estimators
}

\author{
Min Zhang*† and Douglas E. Schaubel
}

\begin{abstract}
In settings where a randomized trial is infeasible, observational data are frequently used to compare treatmentspecific survival. The average causal effect (ACE) can be used to make inference regarding treatment policies on patient populations, and a valid ACE estimator must account for imbalances with respect to treatment-specific covariate distributions. One method through which the ACE on survival can be estimated involves appropriately averaging over Cox-regression-based fitted survival functions. A second available method balances the treatment-specific covariate distributions through inverse probability of treatment weighting and then contrasts weighted nonparametric survival function estimators. Because both methods have their advantages and disadvantages, we propose methods that essentially combine both estimators. The proposed methods are double robust, in the sense that they are consistent if at least one of the two working regression models (i.e., logistic model for treatment and Cox model for death hazard) is correct. The proposed methods involve estimating the ACE with respect to restricted mean survival time, defined as the area under the survival curve up to some prespecified time point. We derive and evaluate asymptotic results through simulation. We apply the proposed methods to estimate the ACE of donation-after-cardiac-death kidney transplantation with the use of data obtained from multiple centers in the Netherlands. Copyright @ 2012 John Wiley \& Sons, Ltd.
\end{abstract}

Keywords: average causal effect; Cox regression; double-robust estimator; inverse weighting; right censoring; restricted mean lifetime

\section{Introduction}

Observational data are frequently used to compare treatment-specific survival in settings where a randomized clinical trial is infeasible. Even in cases where a randomized trial to compare treatments is feasible, observational studies may be an attractive alternative because much greater sample sizes can be obtained at considerably less cost and effort. Methods applicable to observational data include those which accommodate imbalances with respect to the treatment-specific distributions of pretreatment patient characteristics. Covariate adjustment may also be desired for randomized trials, to adjust for chance imbalances in adjustment factors and perhaps to increase precision.

Despite the value of observational studies, the randomized trial rightfully serves as the gold standard. This has important implications from at least two angles. First, in analyzing observational data, one is motivated to compute an estimator whose target (e.g., difference in treatment-specific means) would be obtained in the setting where treatment was randomized. Second, there is an incentive to estimate quantities that would be estimated in the context of a randomized trial. For example, in the case of censored survival data, the analysis of data from a randomized trial would likely consist of plots of treatment-specific Kaplan-Meier or Nelson-Aalen survival curves. Survival probability is easily intuited by nonstatisticians. A related measure is the area under the survival curve. In particular, if $T$ represents failure time with survival function, $P(T>t)=S(t)$, then mean survival time is equal to the area under the entire survival curve, given by $E[T]=\int_{0}^{\infty} S(t) \mathrm{d} t$. The most popular methods of estimating $S(t)$ in settings where covariate adjustment is unnecessary are nonparametric; that is, the aforementioned Kaplan-Meier [1] and Nelson-Aalen methods. In cases where covariate effects are modeled, the Cox [2] model (a semiparametric approach) has dominated the hazard regression applications in the 
biomedical literature almost since its inception. The nonparametric aspects of each of the three aforementioned methods result in inference that is restricted to the $(0, \tau]$ time interval, where $\tau$ is the maximum observation time. Although mean survival time may be of most inherent interest, because inference is on a restricted range anyway, restricted mean lifetime, $E[\min \{T, L\}]=\int_{0}^{L} S(t) \mathrm{d} t$, is a useful and practical alternative metric; for example, see [3-9]. Restricted mean lifetime has a straightforward interpretation (i.e., expected number of time units lived out of the next $L$ ) and is the measure of interest in this report.

With respect to restricted mean lifetime, the average causal effect (ACE) is the area between the average treatment-specific survival curves (out to $t=L$ ), with the averaging (for both treatments) being with respect to the marginal covariate distribution. As will be explicitly developed later, this quantity is the same as the area between the unadjusted treatment-specific survival curves in the setting of a randomized study. There are different ways to estimate the pertinent treatment-specific average survival functions. Various authors have advocated fitting Cox models, then explicitly averaging over fitted survival curves (e.g., $[3,5,10])$. An alternative method involves inverse probability of treatment weighting (IPTW) [11-15]. In IPTW, each subject is weighted by the inverse of the probability of being assigned the treatment they actually received. The weighted treatment-specific samples have a covariate distribution that equals that of the margin (across both treatments). Hence, differences between integrated IPTW versions of nonparametric estimators serve as estimators of the ACE of restricted mean lifetime. For example, Hubbard et al. [16] developed IPTW methods to contrast survival curves, whereas Wei [15] proposed various measures based on $S(t)$ with the use of the IPTW method, including restricted mean lifetime. Instead of inverse probability weighting, a popular alternative is to match by the probability of being treated $[17,18]$, which we do not consider in this article.

In this report, we propose a semiparametric double-robust estimator of the ACE on restricted mean lifetime. The proposed method can be viewed as a hybrid of the approaches of Chen and Tsiatis [10] and Wei [15]. A logistic model is assumed for treatment assignment, and a Cox model is used for the death hazard conditional on treatment and the adjustment covariates; both are working models. The proposed methods are double robust in the sense that consistent estimation of the ACE is obtained if at least one of the two working models is correct [19-21].

The data that motivate our methods originate from a multicenter study of kidney transplant patients from the Netherlands [22]. The practice of transplanting kidneys from deceased donors following cardiac death (so-called donation-after-cardiac-death (DCD) kidneys) is controversial. The practice happens to be much more frequent in the Netherlands than other parts of the world (e.g., the USA). We sought to estimate the ACE of DCD kidney transplantation versus its alternative, which we refer to collectively as non-DCD transplantation.

We organize the remainder of this report as follows. In the next section, we formalize the ideas outlined previously and describe the proposed methods and corresponding asymptotic properties. We assess the finite-sample applicability of the procedures through simulation in Section 3. We then use the proposed methods in Section 4 to analyze the kidney transplant data described previously. Section 5 concludes the report with some discussion.

\section{Method}

Suppose we are interested in comparing two groups, with group denoted by $A$ (with $A=0$ or 1 ) in terms of the mean of the restricted lifetime up to time $L$. If we denote the survival time by $T$, then the restricted lifetime is defined as $\min (T, L)$ and the restricted mean lifetime can be represented as $E\{\min (T, L)\}$. In the setup we consider in this article, treatment groups are not randomized, and therefore some sort of adjustment for imbalance in baseline covariates, $Z$, is required. As in almost all studies involving time to an event, survival time is subject to right censoring, denoted by $C$. We assume that censoring is conditionally independent of death time given treatment; that is, $T \Perp C \mid A$, an assumption that we discuss further in Section 5. We define the observed possibly censored lifetime as $U=\min (T, C)$ and the indicator for not being censored as $\Delta=I(T \leqslant C)$. The observed data for each subject $i$ are $\left(A_{i}, Z_{i}, U_{i}, \Delta_{i}\right)$, which are assumed to be independent and identically distributed across $i=1, \ldots, n$. We denote the observed counting process of event and the at-risk process by $N_{i}(t)=I\left(U_{i} \leqslant t, \Delta_{i}=1\right)$ and $Y_{i}(t)=I\left(U_{i} \geqslant t\right)$, respectively. For simplicity of presentation, we define $A_{i j}=I\left(A_{i}=j\right)$, $N_{i j}(t)=A_{i j} N_{i}(t)$, and $Y_{i j}(t)=A_{i j} Y_{i}(t)$.

The quantity that we would like to infer in the comparison of two treatments (the ACE) is the difference in restricted mean lifetimes had all subjects in the population received treatment $A=1$ as opposed 
to that had all subjects received $A=0$. Specifically, denoting the potential (counterfactual) lifetime of a subject if, possibly contrary to fact, he or she received treatment $A=j$ by $T^{j}, j=0,1$, the restricted mean lifetime for treatment $A=j$ is $\mu_{j}=E\left\{\min \left(T^{j}, L\right)\right\}$, and the treatment effect can be contrasted through the difference, $\delta=\mu_{1}-\mu_{0}$. It can be shown that restricted mean lifetime can be represented as the area under the survival curve up to $L, \mu_{j}=\int_{0}^{L} S^{j}(t) \mathrm{d} t$, where $S^{j}(t)$ is the marginal survival function of $T^{j}$. Therefore, treatment-specific restricted mean lifetimes and their difference represent a cumulative measure of treatment effects. Throughout this article, we will make the stable unit treatment value assumption $[23,24]$, which assumes that there is no interference between subjects and that the observed survival time $T$ for a subject receiving treatment $A=j$ is equal to his or her potential lifetime under the treatment $j$; that is, $T=A T^{1}+(1-A) T^{0}$. This assumption usually holds in a study with no interference between subjects, as in the application we will consider. However, this assumption may not hold, for example, in studies involving infectious disease as subjects interfere with each other and a subject's potential response under one treatment may be influenced by treatments other subjects receive.

The ACE, $\delta$, is defined in terms of potential outcomes, which are not observed for all subjects. Nevertheless, inference on the hypothetical quantity $\delta$ has to be based on observed data. In observational data, the distribution of baseline covariates $Z$ among subjects in one group is possibly different from that in the other group; or, equivalently, $Z$ is not independent of $A$. If these covariates also predict potential survival times, that is, $Z$ is not independent of $\left(T^{0}, T^{1}\right)$, then both treatment difference and differences in $Z$ contribute to the observed difference in survival times between two groups; that is, the effect of treatment on survival is confounded by imbalance in covariates. Put another way, treatment assignment is not independent of the potential lifetimes due to their mutual correlation with covariates $Z$, also referred to as confounders. This nonindependence introduces some difficulty in making causal inference based on observational data, in contrast with randomized study where by design treatment assignment is independent of potential lifetimes. A key assumption that allows causal inference on observational data possible is that, conditional on $Z$, treatment assignment can be viewed as random in the sense that it is independent of potential lifetimes; that is, $A \Perp\left(T^{0}, T^{1}\right) \mid Z$. This assumption, required by both the Chen and Tsiatis [10] method and the IPTW method of Wei [15], is referred to as the 'strong ignorability' assumption of treatment [17] or the 'no unmeasured confounders' assumption [12]. Note that this condition is also well studied in the economics and social science literature; for example, $[25,26]$. This assumption states that the dependence of treatment assignment on the potential outcomes can be completely eliminated by the observed variables $Z$. This assumption cannot be tested statistically and can only be justified based on knowledge on the subject matter. In medical applications, treatment decisions made by patients or their caregivers are usually based on information available at the time of the decision making, such as demographics, comorbidities, severity, and past treatments of the subjects. If such information is also captured in the data at hand, then this assumption is plausible.

In the next two paragraphs, we describe the Chen and Tsiatis method [10] and the IPTW method of Wei [15], in order to later establish the relationship between each of these two methods and our proposed method. Much of the notation introduced here will be needed later in the development of the proposed method. The method of Chen and Tsiatis [10] removes confounding by first estimating the treatment effect conditional on covariates $Z$. This part is straightforward because, conditional on $Z$, treatment $A$ can be viewed as randomly assigned. The conditional treatment effect measures the difference in survival had subjects with covariates $Z=z$ been assigned to $A=1$ versus $A=0$, because $f(T \mid A=j, Z=z)=f\left(T^{j} \mid A=j, Z=z\right)=f\left(T^{j} \mid Z=z\right)$ for $j=0,1$, where $f$ denotes 'distribution of' and the two equalities are due to the stable unit treatment value assumption and no unmeasured confounders assumptions, respectively. Note that this result implies $P\left(T^{j}>t \mid Z\right)=P(T>t \mid A=j, Z)$, which relates the conditional survival functions of potential lifetimes to those of the observed lifetimes. Next, the ACE of $A$ can be estimated by the average of the conditional effects across the distribution of $Z$. Specifically, the Chen and Tsiatis method [10] posits treatment-stratified Cox models [2] for $T$ given $(A, Z)$,

$$
\lambda_{i j}(t) \equiv \lambda\left(t \mid A_{i}=j, Z_{i}\right)=\lambda_{0 j}(t) e^{\beta_{j}^{T} Z_{i}}, \quad j=0,1,
$$

where $\lambda(t \mid A, Z)$ denotes the conditional hazard function given $A$ and $Z$, and $\lambda_{0 j}(t)$ are unspecified treatment-specific baseline hazard functions. Inference on this model can be carried out by standard survival analysis techniques. For example, $\beta_{j}$ can be estimated by the maximum partial likelihood 


\section{Statistics}

estimator, $\widehat{\beta}_{j}[2,27]$, whereas the baseline cumulative hazard function $\Lambda_{0 j}(t) \equiv \int_{0}^{t} \lambda_{0 j}(u) \mathrm{d} u$ can be consistently estimated by the Breslow estimator [28], denoted by $\widehat{\Lambda}_{0 j}(t) \equiv \int_{0}^{t} \frac{\sum_{i=1}^{n} \mathrm{~d} N_{i j}(t)}{\sum_{i=1}^{n} Y_{i j}(t) \exp \left(\widehat{\beta}_{j}^{T} Z_{i}\right)}$. The treatment effect (difference in restricted mean lifetimes) conditional on $Z$ can be estimated by $\int_{0}^{L}\left\{e^{-\widehat{\Lambda}_{i 1}(t)}-e^{-\widehat{\Lambda}_{i 0}(t)}\right\} \mathrm{d} t$, where $e^{-\widehat{\Lambda}_{i j}(t)}$ estimates the conditional survival function $P\left(T_{i}>t \mid A_{i}=j, Z_{i}\right)$, with $\widehat{\Lambda}_{i j}(t)=e^{\widehat{\beta}_{j}^{T} Z_{i}} \widehat{\Lambda}_{0 j}(t)$. Finally, the average causal treatment effect $\delta$ is estimated by averaging the conditional treatment effects across all $Z_{i}$ for $i=1, \ldots, n$; that is, $\tilde{\delta}=n^{-1} \sum_{i=1}^{n} \int_{0}^{L}\left\{e^{-\widehat{\Lambda}_{i 1}(t)}-e^{-\widehat{\Lambda}_{i 0}(t)}\right\} \mathrm{d} t$. Note that $n^{-1} \sum_{i=1}^{n} e^{-\widehat{\Lambda}_{i j}(t)}$ estimates the marginal survival function $S^{j}(t)$, and therefore $\tilde{\delta}$ is also the integrated difference in estimated marginal survival functions.

Instead of going through the treatment effect (and treatment-specific survival functions) conditional on covariates, a different strategy is to estimate the average treatment effect directly through weighted nonparametric estimators. In particular, the IPTW method [15] removes confounding by building up the whole population that could have received the treatment, say, $A=j$, by inverse weighting the individuals in group $j$ with the probability of being in that group conditional on covariates. For example, if a subject in treatment $j$ has covariates $Z=z$ and the probability of receiving the treatment $P(A=j \mid Z=z)$, then this subject actually represents $1 / P(A=j \mid Z=z)$ individuals in the population of interest that could have received the treatment and inverse weighting by this probability builds up the whole population. Specifically, as marginal survival function can be estimated through the Nelson-Aalen estimator of cumulative hazard function, the IPTW estimator for the marginal cumulative hazard function of $T^{j}, \Lambda_{j}(t)$, can be viewed as a weighted Nelson-Aalen estimator; that is,

$$
\widehat{\Lambda}_{j}^{\mathrm{IPTW}}(t)=\int_{0}^{t} \frac{\sum_{i=1}^{n} w_{i j}(\widehat{\theta}) \mathrm{d} N_{i}(t)}{\sum_{i=1}^{n} w_{i j}(\widehat{\theta}) Y_{i}(t)}
$$

where $w_{i j}(\widehat{\theta})=I\left(A_{i}=j\right) / p_{i j}(\widehat{\theta})$ and $p_{i j}(\widehat{\theta})$ is an estimator of $P\left(A_{i}=j \mid Z_{i}\right)$. Note that if the weights, $w_{i j}$, are set to 1 , then (2) reduces the usual Nelson-Aalen estimator of the cumulative hazard function of $T$. If, instead, $p_{i j}(\widehat{\theta})$ is set to 1 , then (2) reduces to the Nelson-Aalen estimator conditional on each treatment. The probability $P\left(A_{i}=j \mid Z_{i}\right)$ can be estimated by fitting a logistic regression model, which assumes

$$
\operatorname{logit}\left\{P\left(A_{i}=1 \mid Z_{i}\right)\right\}=\theta^{T} X_{i},
$$

where $X_{i}$ includes an intercept and (possibly transformed) elements of $Z_{i}$ and $\operatorname{logit}(u)=\log \{u /(1-u)\}$.

The Chen and Tsiatis [10] method builds models for survival time, and the resulting estimators for $S^{j}(t), j=0,1$, and $\delta$ are consistent for the true ACE if the assumed model (1) is correct. The IPTW method builds a logistic model for treatment assignment, and the resulting estimators are consistent if the assumed logistic model (3) is correct. In our proposed method, we propose to build models for both the treatment assignment and survival time. The strategy is to attempt to either model the treatment assignment correctly, allowing one to balance the distribution of covariates between treatments, or to model the survival process correctly. If at least one of the models is correct, then the ACE can be estimated consistently. Therefore, in the following discussions, we will refer to models (1) and (3) as working models as they are not necessarily believed to be true. As the IPTW method, the proposed method estimates treatment effect through estimating the marginal cumulative hazard functions, $\Lambda_{j}(t)$. Specifically, the proposed estimator for $\Lambda_{j}(t)$ is

$$
\widehat{\Lambda}_{j}(t)=\int_{0}^{t} \frac{n^{-1} \sum_{i=1}^{n} e^{-\widehat{\Lambda}_{i j}(u)} \mathrm{d} \widehat{\Lambda}_{i j}(u)+n^{-1} \sum_{i=1}^{n}\left[w_{i j}(\widehat{\theta})\left\{e^{\widehat{\Lambda}_{j}^{C}(u)} \mathrm{d} N_{i j}(u)-e^{-\widehat{\Lambda}_{i j}(u)} \mathrm{d} \widehat{\Lambda}_{i j}(u)\right\}\right]}{n^{-1} \sum_{i=1}^{n} e^{-\widehat{\Lambda}_{i j}(u)}+n^{-1} \sum_{i=1}^{n}\left[w_{i j}(\widehat{\theta})\left\{e^{\widehat{\Lambda}_{j}^{C}(u)} Y_{i j}(u)-e^{-\widehat{\Lambda}_{i j}(u)}\right\}\right]},
$$


where $\widehat{\Lambda}_{j}^{C}(u)$ is the Nelson-Aalen estimator of the treatment-specific cumulative hazard function (given $A=j$ ) of $C$,

$$
\widehat{\Lambda}_{j}^{C}(t)=\int_{0}^{t} \frac{\sum_{i=1}^{n} \mathrm{~d} N_{i j}^{C}(u)}{\sum_{i=1}^{n} Y_{i j}(u)},
$$

with $N_{i j}^{C}(t)=A_{i j} I\left(U_{i} \leqslant t, \Delta_{i}=0\right)$. Consequently, one can estimate $S^{j}(t)$ by $\widehat{S}^{j}(t)=e^{-\widehat{\Lambda}_{j}(t)}$, and $\mu_{j}$ by $\widehat{\mu}_{j}=\int_{0}^{L} \widehat{S}^{j}(u) \mathrm{d} u$. Finally, the proposed estimator for $\delta$ is given by $\widehat{\delta}=\widehat{\mu}_{1}-\widehat{\mu}_{0}$.

Before introducing the main theorem regarding asymptotic properties of the proposed estimators, let us first heuristically explain how to understand this method and why it is expected to possess the double-robust property. Note that Kang and Schafer [20] provided an exposition of double-robust estimators in the context of continuous outcomes. As $\Lambda_{j}(t)$ can be expressed as $-\int_{0}^{t} S^{j}(u)^{-1} \mathrm{~d} S^{j}(u)$, our proposed method estimates $\Lambda_{j}(t)$ by estimating $S^{j}(u)$ and $-\mathrm{d} S^{j}(u)$, respectively. Specifically, the denominator inside the integral in (4) estimates $S^{j}(u)$ and the numerator estimates $-\mathrm{d} S^{j}(u)$. Considering the denominator, the first term, $n^{-1} \sum_{i=1}^{n} e^{-\widehat{\Lambda}_{i j}(u)}$, is the Chen and Tsiatis estimator of $S^{j}(u)$ and, as explained earlier, is an average of estimators of the conditional survival functions $P\left(T_{i}>u \mid A_{i}=j, Z_{i}\right)$ across $i=1, \ldots, n$. Suppose hypothetically that every subject in the population received treatment $j$ and, in addition, that censoring does not exist. In this setting, $I\left(T_{i} \geqslant u\right)$ would serve as the response, such that the residual for each subject at time $u$, in the fit of model (1), is given by $I\left(T_{i} \geqslant u\right)-e^{-\widehat{\Lambda}_{i j}(u)}$. If the model for survival time is correctly specified, on average the residuals are close to zero; otherwise, the average of residuals estimates the bias of the Chen and Tsiatis estimator. In reality, because the subjects who actually received treatment $j$ are not representative of the whole population as a result of lack of randomization, the average of residuals among those who actually received $A=j$ does not directly estimate the bias. Applying the idea of the IPTW method, it is easy to see that the bias can be consistently estimated from residuals on those who are actually in group $j$ by inverse weighting their contributions by the corresponding probability of being in group $j$. In addition, in reality, even for subjects in group $j, I\left(T_{i} \geqslant u\right)$, and correspondingly the residuals, are not observed for all of them because of censoring, and instead one only observes $Y_{i}(u)$. A solution to this is that one further weights $Y_{i}(u)$ by the probability of not being censored. Therefore, the bias can be estimated by the second term in the denominator, that is, $n^{-1} \sum_{i=1}^{n} w_{i j}(\widehat{\theta})\left\{e^{\widehat{\Lambda}_{j}^{C}(u)} Y_{i}(u)-e^{-\widehat{\Lambda}_{i j}(u)}\right\}$, where $e^{\widehat{\Lambda}_{j}^{C}(u)}$ estimates $P\left(C_{i}>u \mid A_{i}\right)^{-1}$, the inverse probability of remaining uncensored as of time $u$ for group $A_{i}=j$. To summarize, if model (1) is correct, the first term of the denominator estimates $S^{j}(u)$ and the second term estimates zero; if model (1) is possibly wrong but model (3) is correct, then the second term estimates the bias of the first term and again the denominator consistently estimates $S^{j}(u)$. Similarly, one can apply the same idea to the numerator, where the first term can be viewed as an estimator for $-\mathrm{d} S^{j}(u)$ and the second term is either estimator of zero or the bias of the first term. As a result, the proposed estimator (4) is expected to be consistent for the true ACE if at least one of the working models is correct.

We point out that the denominator of (4) is itself a double-robust estimator of $S^{j}(u)$. In our proposed method, we do not use it directly because of the following considerations. The denominator can be written equivalently as $n^{-1} \sum_{i=1}^{n}\left[w_{i j}(\widehat{\theta}) e^{\widehat{\Lambda}_{j}^{C}(u)} Y_{i j}(u)\right]+n^{-1} \sum_{i=1}^{n}\left[\left\{1-w_{i j}(\widehat{\theta})\right\} e^{-\widehat{\Lambda}_{i j}(u)}\right]$, which can be viewed as an augmented IPTW estimator of $S^{j}(u)$ with the first term being an IPTW estimator and the second term as the augmentation term [29]. From this perspective, the denominator builds upon the idea that $E\left\{Y_{i}(t) / P\left(C_{i}>t\right)\right\}=P\left(T_{i}>t\right)$ in a one-sample setting. Few practitioners would use this method in the one-sample setting, in part because the resulting survival curve is not monotone. The two most popular estimators in the one-sample setting are the Kaplan-Meier and Nelson-Aalen estimators. Our method builds on the latter (and the Kaplan-Meier estimator is asymptotically equivalent to the Nelson-Aalen estimator). One can interpret our estimator as a modified Nelson-Aalen estimator, with the modifications being to incorporate adjustment covariates and double robustness. In addition, simulation studies show that using the denominator to estimate $S^{j}(u)$ directly and then to estimate $\mu_{j}$ may lead to considerably more bias than the proposed method in finite samples.

We summarize the asymptotic properties of the proposed estimators for $\mu_{j}$ and $\delta$ with the following theorem; we outline the proof in Appendix A. 


\section{Theorem 1}

Under conditions (a)-(f) listed in Appendix A, as $n \rightarrow \infty$, if at least one of the working models specified in (1) and (3) is correct, then $\widehat{\mu}_{j}$ converges in probability to $\mu_{j}$ and $n^{\frac{1}{2}}\left(\widehat{\mu}_{j}-\mu_{j}\right)$ is asymptotically normal with mean zero and variance $E\left(\phi_{i j}^{2}\right)$, where $\phi_{i j}=-\int_{0}^{L} S^{j}(u) \varphi_{i j}(u) \mathrm{d} u$,

$$
\begin{aligned}
\varphi_{i j}(t)= & B_{j}^{T}\left(t ; \beta_{j}^{*}, \theta^{*}\right) V^{-1}\left(\theta^{*}\right) X_{i}\left\{A_{i j}-p_{i j}\left(\theta^{*}\right)\right\}+F_{j}^{T}\left(t ; \beta_{j}^{*}\right) \Omega_{j}^{-1}\left(\beta_{j}^{*}\right) U_{i j}\left(\beta_{j}^{*}\right) \\
& +\int_{0}^{t} G_{j}\left(u, t ; \beta_{j}^{*}, \theta\right) \frac{\mathrm{d} M_{i j}^{*}(u)}{r_{j}^{(0)}\left(u ; \beta_{j}\right)}+\int_{0}^{t} H_{j}\left(u, t ; \beta_{j}^{*}, \theta^{*}\right) \frac{\mathrm{d} M_{i j}^{C}(u)}{E\left\{Y_{i j}(u)\right\}} \\
& +\int_{0}^{t} \frac{w_{i j}\left(\theta^{*}\right) e^{\Lambda_{j}^{C}(u)} \mathrm{d} M_{i j}^{\dagger}(u)-\left\{w_{i j}\left(\theta^{*}\right)-1\right\} e^{-\Lambda_{i j}^{*}(u)}\left\{\mathrm{d} \Lambda_{i j}^{*}(u)-\mathrm{d} \Lambda_{j}(u)\right\}}{D_{j}\left(u ; \beta_{j}^{*}, \theta^{*}\right)},
\end{aligned}
$$

$\theta^{*}$ and $\beta_{j}^{*}$ are the asymptotic limiting values of $\widehat{\theta}$ and $\widehat{\beta}_{j}$, respectively, which may or may not equal to the respective true values, depending on whether the corresponding assumed model is correct, and $\mathrm{d} M_{i j}^{\dagger}(u)=\mathrm{d} N_{i j}(u)-Y_{i j}(u) \mathrm{d} \Lambda_{j}(u)$, with $B_{j}\left(t ; \beta_{j}^{*}, \theta^{*}\right), F_{j}\left(t ; \beta_{j}^{*}, \theta^{*}\right), G_{j}\left(u, t ; \beta_{j}^{*}, \theta^{*}\right)$, $H_{j}\left(u, t ; \beta_{j}^{*}, \theta^{*}\right)$ defined in Appendix A. In addition, under the same conditions, $\widehat{\delta}$ converges in probability to $\delta$ and $n^{\frac{1}{2}}(\widehat{\delta}-\delta)$ is asymptotically normal with mean zero and variance $E\left(\phi_{i 1}-\phi_{i 0}\right)^{2}$.

In the aforementioned theorem, $\varphi_{i j}$ and $\phi_{i j}$ seem complicated, and this is because it is stated without explicitly assuming which working model is correctly specified. If one or both of the working models are correctly specified, some of the terms in $\varphi_{i j}(t)$ and $\phi_{i j}$ are identically zero. For example, if the model (1) is correct, then $B_{j}\left(t ; \beta_{j}^{*}, \theta^{*}\right)$ is equal to zero, and if the model (3) is the true model, then $F_{j}\left(t ; \beta_{j}^{*}, \theta^{*}\right)$ and $G_{j}\left(u, t ; \beta_{j}^{*}, \theta^{*}\right)$ are identically zero. Variance for $\widehat{\mu}_{j}$ and $\widehat{\delta}$ can be consistently estimated through $n^{-1} \sum_{i=1}^{n} \widehat{\phi}_{i j}^{2}$ and $n^{-1} \sum_{i=1}^{n}\left(\widehat{\phi}_{i 1}-\widehat{\phi}_{i 0}\right)^{2}$, respectively, where $\widehat{\phi}_{i j}$ is obtained by replacing limiting values in $\widehat{\phi}_{i j}$ with their empirical counterparts. Although $\widehat{\phi}_{i j}$ seems complicated, variance estimators can actually be computed fast. SAS code illustrating the implementation of the proposed methods and the variance estimators are available at http://www-personal.umich.edu/ mzhangst.

\section{Simulation studies}

In this section, we report results from simulation studies to evaluate the finite sample properties of the proposed method. Results are based on 1000 Monte Carlo data sets with a sample size of $n=600$.

In our simulated data, baseline covariates, $Z_{1}, Z_{2}, Z_{3}$, are generated as normal with zero mean and unit variance, and the correlation between $Z_{1}$ and $Z_{3}$ is 0.2 . Each covariate is truncated at -4 and 4 to be consistent with the regularity conditions listed in the Appendix. We then generated treatment indicator $A$, according to a logistic regression model with $\operatorname{logit}\{P(A=1 \mid Z)\}=-0.5 Z_{1}-0.5 Z_{2}$. Lifetime $T$ was generated as exponential with rate $\exp \left(-2.5-1.5 Z_{1}-Z_{2}-0.7 Z_{3}\right)$ for treatment $A=0$ and $\exp \left(-3-Z_{1}-0.9 Z_{2}-Z_{3}\right)$ for $A=1$, respectively. Note that covariates $Z_{1}$ and $Z_{2}$ predict both treatment assignment and survival time; therefore, they serve as confounders. Finally, we generated censoring time $C$ as exponential with rate $\exp (-4.5)$, which leads to approximately $25 \%$ censoring.

We compare the proposed method with the other methods introduced previously: the method of Chen and Tsiatis [10], where one models the relationship of survival time to covariates by treatment-specific Cox models; and the IPTW method of Wei [15] wherein one instead models the treatment assignment with covariates using a logistic regression model. We evaluate each of the three estimators under settings where the assumed models for survival time and treatment assignment are both correct or both incorrect or only one of them is correct. Specifically, for the $T \mid A, Z$ model used in both the proposed and Chen and Tsiatis [10] methods, we fit the correct model with the use of covariates $\left(Z_{1}, Z_{2}, Z_{3}\right)$ for each treatment, whereas we fit the incorrect model with the use of $\left(Z_{1}, Z_{3}\right)$. For the $A \mid Z$ model used in the proposed and the IPTW methods, we fit the correct model with the use of $\left(Z_{1}, Z_{2}\right)$, whereas we fit the incorrect model with the use of $Z_{1}$ only.

We estimated restricted mean lifetimes and their difference with $L$ set to 10 and 20. Tables I and II summarize results for estimating $\mu_{1}$ and $\delta$, respectively, and results for $\mu_{0}$ are very similar and therefore 
Table I. Estimation of restricted mean lifetime for $A=1$.

\begin{tabular}{|c|c|c|c|c|c|c|c|}
\hline Method & $T$ model & $A$ model & True & Bias & ESD & ASE & $\mathrm{CP}$ \\
\hline \multicolumn{8}{|c|}{$L=10$} \\
\hline \multirow[t]{4}{*}{ Proposed } & $\mathrm{T}$ & $\mathrm{T}$ & 6.849 & 0.002 & 0.201 & 0.193 & 0.942 \\
\hline & $\mathrm{T}$ & $\mathrm{F}$ & & -0.002 & 0.201 & 0.191 & 0.936 \\
\hline & $\mathrm{F}$ & $\mathrm{T}$ & & 0.002 & 0.205 & 0.196 & 0.937 \\
\hline & $\mathrm{F}$ & $\mathrm{F}$ & & -0.302 & 0.218 & 0.207 & 0.696 \\
\hline \multirow[t]{4}{*}{ Denominator } & $\mathrm{T}$ & $\mathrm{T}$ & & 0.028 & 0.205 & & \\
\hline & $\mathrm{T}$ & $\mathrm{F}$ & & 0.028 & 0.203 & & \\
\hline & $\mathrm{F}$ & $\mathrm{T}$ & & 0.029 & 0.209 & & \\
\hline & $\mathrm{F}$ & $\mathrm{F}$ & & -0.275 & 0.220 & & \\
\hline \multirow[t]{2}{*}{ IPTW } & & $\mathrm{T}$ & & 0.001 & 0.212 & 0.204 & 0.938 \\
\hline & & $\mathrm{F}$ & & -0.304 & 0.227 & 0.217 & 0.715 \\
\hline \multirow[t]{2}{*}{ Chen and Tsiatis } & $\mathrm{T}$ & & & 0.006 & 0.194 & 0.182 & 0.929 \\
\hline & $\mathrm{F}$ & & & -0.378 & 0.212 & 0.202 & 0.535 \\
\hline \multicolumn{8}{|c|}{$L=20$} \\
\hline \multirow[t]{4}{*}{ Proposed } & $\mathrm{T}$ & $\mathrm{T}$ & 11.488 & 0.017 & 0.426 & 0.418 & 0.941 \\
\hline & $\mathrm{T}$ & $\mathrm{F}$ & & 0.008 & 0.422 & 0.410 & 0.938 \\
\hline & $\mathrm{F}$ & $\mathrm{T}$ & & 0.019 & 0.436 & 0.424 & 0.938 \\
\hline & $\mathrm{F}$ & $\mathrm{F}$ & & -0.686 & 0.453 & 0.438 & 0.652 \\
\hline \multirow[t]{4}{*}{ Denominator } & $\mathrm{T}$ & $\mathrm{T}$ & & 0.071 & 0.438 & & \\
\hline & $\mathrm{T}$ & $\mathrm{F}$ & & 0.071 & 0.427 & & \\
\hline & $\mathrm{F}$ & $\mathrm{T}$ & & 0.073 & 0.447 & & \\
\hline & $\mathrm{F}$ & $\mathrm{F}$ & & -0.630 & 0.457 & & \\
\hline \multirow{2}{*}{ IPTW } & & $\mathrm{T}$ & & 0.016 & 0.454 & 0.449 & 0.946 \\
\hline & & $\mathrm{F}$ & & -0.690 & 0.474 & 0.463 & 0.684 \\
\hline \multirow[t]{2}{*}{ Chen and Tsiatis } & $\mathrm{T}$ & & & 0.021 & 0.410 & 0.395 & 0.938 \\
\hline & $\mathrm{F}$ & & & -0.810 & 0.438 & 0.426 & 0.533 \\
\hline
\end{tabular}

$T$ model indicates whether the model for $T$ is true or false; $A$ model indicates whether the model for $A$ is true or false. Bias is the Monte Carlo bias; ESD is the Monte Carlo standard deviation of estimates; ASE is the Monte Carlo average of estimated standard errors; and CP is the coverage probability of nominal $95 \%$ Wald confidence intervals.

\begin{tabular}{|c|c|c|c|c|c|c|c|}
\hline Method & $T$ model & $A$ model & True & Bias & ESD & ASE & $\mathrm{CP}$ \\
\hline \multicolumn{8}{|c|}{$L=10$} \\
\hline \multirow[t]{4}{*}{ Proposed } & $\mathrm{T}$ & $\mathrm{T}$ & 0.871 & -0.010 & 0.220 & 0.222 & 0.945 \\
\hline & $\mathrm{T}$ & $\mathrm{F}$ & & -0.022 & 0.217 & 0.228 & 0.953 \\
\hline & $\mathrm{F}$ & $\mathrm{T}$ & & -0.009 & 0.229 & 0.230 & 0.953 \\
\hline & $\mathrm{F}$ & $\mathrm{F}$ & & -0.686 & 0.260 & 0.259 & 0.245 \\
\hline \multirow[t]{2}{*}{ IPTW } & & $\mathrm{T}$ & & -0.013 & 0.248 & 0.256 & 0.960 \\
\hline & & $\mathrm{F}$ & & -0.690 & 0.279 & 0.285 & 0.329 \\
\hline \multirow[t]{2}{*}{ Chen and Tsiatis } & $\mathrm{T}$ & & & -0.004 & 0.208 & 0.204 & 0.943 \\
\hline & $\mathrm{F}$ & & & -0.726 & 0.256 & 0.256 & 0.188 \\
\hline \multicolumn{8}{|c|}{$L=20$} \\
\hline \multirow[t]{4}{*}{ Proposed } & $\mathrm{T}$ & $\mathrm{T}$ & 1.682 & -0.009 & 0.454 & 0.453 & 0.946 \\
\hline & $\mathrm{T}$ & $\mathrm{F}$ & & -0.031 & 0.449 & 0.465 & 0.956 \\
\hline & $\mathrm{F}$ & $\mathrm{T}$ & & -0.005 & 0.470 & 0.467 & 0.953 \\
\hline & $\mathrm{F}$ & $\mathrm{F}$ & & -1.450 & 0.537 & 0.533 & 0.208 \\
\hline \multirow[t]{2}{*}{ IPTW } & & $\mathrm{T}$ & & -0.013 & 0.510 & 0.518 & 0.956 \\
\hline & & $\mathrm{F}$ & & -1.460 & 0.580 & 0.586 & 0.295 \\
\hline \multirow[t]{2}{*}{ Chen and Tsiatis } & $\mathrm{T}$ & & & 0.002 & 0.420 & 0.417 & 0.950 \\
\hline & $\mathrm{F}$ & & & -1.500 & 0.518 & 0.520 & 0.167 \\
\hline
\end{tabular}

Entries as in Table I. 


\begin{tabular}{|lcccc|}
\hline \multicolumn{4}{|l}{ Table III. Estimation of 5-year restricted mean lifetimes for DCD and } \\
non-DCD kidney recipients and their difference. \\
Method & $\widehat{\mu}_{0}$ & $\widehat{\mu}_{1}$ & $\delta$ & $p$-value \\
\hline IPTW of Wei & $4.53(0.057)$ & $4.66(0.068)$ & $0.14(0.088)$ & 0.11 \\
Chen and Tsiatis & $4.50(0.058)$ & $4.66(0.063)$ & $0.16(0.084)$ & 0.06 \\
Proposed & $4.54(0.057)$ & $4.64(0.068)$ & $0.105(0.088)$ & 0.23 \\
\hline
\end{tabular}

Standard error for each estimator is reported in parenthesis, and $p$-value is for comparison of the mean restricted lifetimes between the two groups.

are not reported. Under all scenarios in which at least one of the working models is correctly specified, the proposed estimators perform well, which is consistent with the purported double-robust property of the proposed methods. Specifically, the proposed estimators are approximately unbiased for the true parameters, and the $95 \%$ coverage probabilities achieve the nominal level. In contrast, the Chen and Tsiatis and IPTW estimators perform well when the corresponding assumed model is correct; however, large biases and small coverage probabilities are observed if the assumed model is incorrect.

As mentioned before, the denominator of (4) itself can be used as an estimator of the survival function $S^{j}(u)$, which can be integrated to estimate $\mu_{j}$ as well. Although asymptotically it is also double robust in the sense that it is consistent for the truth when at least one of the working models is correct, our simulation studies show that it has considerable larger bias than the proposed method (Table I).

\section{Application}

We applied the proposed methods to compare survival following deceased-donor kidney transplantation among patients receiving a transplant through DCD versus the remainder (referred to here as non-DCD). Eurotransplant, the Dutch Organ Transplant Registry and the Bureau of Geneaology, provided the data. Specifically, Eurotransplant collected dates of registration on the kidney waiting list and, where applicable, kidney transplantation. The Dutch Organ Transplant Registry provided information regarding donor and recipient characteristics, as well as date of death. Data from the Bureau of Geneaology served as the basis for verifying mortality information.

A total of $n=1139$ patients were included in the analysis; 459 of whom received DCD kidney transplants and 680 non-DCD transplants. The mean age at transplant was approximately 49 years, and there were 88 observed deaths. As indicated previously, the groups being compared were $\operatorname{DCD}(j=1)$ versus non-DCD $(j=0)$. Adjustment covariates included age, sex, vascular disease (as a primary renal diagnosis), panel reactive antibodies, expanded criteria donor, method of first dialysis, and years on dialysis prior to transplant.

Under the proposed method (Table III), mean 5-year post-transplant survival time is estimated to be $\widehat{\mu}_{1}=4.64$ years for the DCD group and $\widehat{\mu}_{0}=4.54$ years for the non-DCD group, for difference of $\widehat{\delta}=0.10$ years $(p=0.23)$. Therefore, there appears to be no difference in 5 -year restricted mean lifetime between recipients of DCD versus non-DCD kidneys. Results were similar based on the method of Chen and Tsiatis [10] and the IPTW approach. In terms of precision, the lowest estimated standard error was from the Chen and Tsiatis [10] method; because this method also estimated the largest difference $\widehat{\delta}$, it also yielded the lowest $p$-value, albeit still nonsignificant $(p=0.06)$. However, the validity of this method requires the Cox model to be correct, unlike the proposed method, which only requires that either the Cox model or the logistic model is correct.

\section{Discussion}

We propose a semiparametric double-robust estimator of the difference in treatment-specific restricted mean survival time. The proposed method uses working models for treatment assignment and the death hazard, and is consistent if at least one of the two working models is correct. We derive and show asymptotic properties of the proposed estimator through simulation to be applicable to practical-sized samples.

We compare our method with two existing methods through which differences in restricted mean lifetimes can be estimated. The method of Chen and Tsiatis fits group-specific Cox models, then 
averages over the fitted values to obtain the ACE. The IPTW method uses inverse probability of treatment weighting to estimate the ACE. Our proposed method can be viewed as a combination of Chen and Tsiatis and the IPTW methods. We obtain consistency if the hazard regression model (from Chen and Tsiatis method) or the group assignment model (used in IPTW method) are correctly specified.

A potential disadvantage of our method is that censoring times are assumed to be conditionally independent of the death times, given only on the treatment indicator. The analogous assumption by Chen and Tsiatis requires conditional independence given both treatment and adjustment covariates, a much less stringent assumption. However, in many observational studies, it is quite reasonable to assume that the adjustment covariates do not predict the censoring hazard. For example, in retrospective cohort studies (e.g., particularly those based on registry or other precollected databases), censoring may be primarily administrative; that is, the date the database was closed, a fixed calendar date that is external to the patients, let alone their adjustment covariate pattern. In the IPTW method of Wei [15], the assumption on the censoring distribution is the same as ours, although the IPTW method does not involve inverse probability of censoring weighting. It should be noted that the IPTW method of Wei has been extended to handle dependent censoring easily, under the 'no-unmeasured-confounders-for-censoring' assumption, by inverse probability of censoring weighting, wherein one models the probability of censoring conditional on baseline and/or time-dependent covariates and modifies the weight function $w_{i j}(\widehat{\theta})$ by further weighting it by the inverse probability of remaining uncensored [30]. However, it is not straightforward to extend the proposed method to accommodate dependent censoring because, in addition to modifying the weight, one needs to modify the second term in both the numerator and the denominator of (4) as well. Therefore, we do not consider this more general assumption on censoring in this article.

The application of the proposed methods implies that 5-year restricted mean post-transplant survival time is no different for patients receiving a kidney transplant through DCD. The importance of this finding is tied to the potential to increase the deceased-donor kidney pool by increasing DCD transplantation. A natural question is whether a difference in restricted mean lifetime would be observed in the presence of a similar study with longer post-transplant follow-up. In fact, in a large percentage of practical settings, it would be preferable to use mean survival time (i.e., without inference being restricted to the $(0, L]$ time interval). The development of robust methods for estimating and contrasting mean lifetime would be valuable.

In this article, we considered estimation of the average causal treatment effect for the entire population of interest. A different but also relevant quantity is the so-called average treatment effect for the treated (ATT) [31], which targets the question of whether the treatment actually worked among treated subjects. In terms of restricted mean lifetimes, the ATT is $E\left\{\min \left(T^{1}, L\right)-\min \left(T^{0}, L\right) \mid A=1\right\}$, and estimating ATT requires a weaker ignorability assumption than the strong ignorability assumption assumed before; that is, $T^{0} \Perp A \mid Z$. We expect that the ideas underlying our proposed method could be extended to estimate the ATT, which would be interesting for future research.

\section{Appendix A.}

\section{A.1. Conditions and preparation results}

We introduce the following notation that will be used in the proof of Theorem 1:

$$
\begin{aligned}
r_{j}^{(d)}(t ; \beta) & =E\left[Y_{i j}(t) Z_{i}^{\otimes d} \exp \left\{\beta^{T} Z_{i}\right\}\right], \quad \bar{z}_{j}(t ; \beta)=\frac{r_{j}^{(1)}(t ; \beta)}{r_{j}^{(0)}(t ; \beta)}, \\
\mathrm{d} \Lambda_{0 j}^{*}(t) & =\frac{E\left\{\mathrm{~d} N_{i j}(t)\right\}}{r_{j}^{(0)}\left(t ; \beta^{*}\right)}, \quad \mathrm{d} \Lambda_{i j}^{*}(t)=\exp \left(\beta_{j}^{* T} Z_{i}\right) \mathrm{d} \Lambda_{0 j}^{*}(t) \\
\mathrm{d} M_{i j}^{*}(t) & =\mathrm{d} N_{i j}(t)-Y_{i j}(t) \mathrm{d} \Lambda_{i j}^{*}(t) \\
\Omega_{j}(\beta) & =\int_{0}^{\tau}\left\{\frac{r_{j}^{(2)}(t ; \beta)}{r_{j}^{(0)}(t ; \beta)}-\bar{z}_{j}(t ; \beta)^{\otimes 2}\right\} E\left\{Y_{i j}(t) \lambda_{i j}(t)\right\} \mathrm{d} t, \\
\text { and } V(\theta) & =E\left[\frac{\exp \left(\theta^{T} X\right) X^{\otimes 2}}{\left\{1+\exp \left(\theta^{T} X\right)\right\}^{2}}\right],
\end{aligned}
$$

for $d=0,1,2$, where for a column vector $a, a^{\otimes 2}=a a^{T}, a^{\otimes 1}=a$, and $a^{\otimes 0}=1$. 
We assume the following regularity conditions for $i=1, \ldots, n$, and $j=0,1$ :

(a) $P\left(U_{i} \geqslant \tau\right)>0$.

(b) $Z_{i}$ is bounded almost surely.

(c) $\Lambda_{0 j}(\tau)<\infty$.

(d) $\beta_{j}^{*}$ is the unique solution to

$$
\int_{0}^{\tau} E\left\{Y_{i j}(t) Z_{i} \lambda_{i j}(t)\right\} \mathrm{d} t-\int_{0}^{\tau} \bar{z}_{j}(t ; \beta) E\left\{Y_{i j}(t) \lambda_{i j}(t)\right\} \mathrm{d} t=0
$$

and $\Omega_{j}\left(\beta^{*}\right)$ is positive definite.

(e) $\theta^{*}$ is the unique maximizer to $E\left\{A \theta^{T} X-\log \left(1+e^{\theta^{T} X}\right)\right\}$, and $V\left(\theta^{*}\right)$ is positive definite.

(f) $P\left(A_{i}=j \mid Z_{i}\right)$ is bounded away from 0 .

With regard to model (1), it was shown that [32], under the assumed regularity conditions, $\widehat{\beta}_{j} \stackrel{p}{\longrightarrow} \beta_{j}^{*}$, with $\beta_{j}^{*}=\beta_{j}$ if model (1) is correct, and $n^{\frac{1}{2}}\left(\widehat{\beta}_{j}-\beta_{j}^{*}\right)$ is asymptotically normal with

$$
n^{\frac{1}{2}}\left(\widehat{\beta}_{j}-\beta_{j}^{*}\right)=\Omega_{j}^{-1}\left(\beta_{j}^{*}\right) n^{-\frac{1}{2}} \sum_{i=1}^{n} U_{i j}\left(\beta_{j}^{*}\right)+o_{p}(1),
$$

where $U_{i j}\left(\beta_{j}^{*}\right)=\int_{0}^{\tau}\left\{Z_{i}-\bar{z}_{j}\left(t ; \beta_{j}^{*}\right)\right\} \mathrm{d} M_{i j}^{*}(t)$. It is then straightforward to show that

$$
\begin{aligned}
n^{\frac{1}{2}}\left\{\widehat{\Lambda}_{i j}(t)-\Lambda_{i j}^{*}(t)\right\}= & K_{i j}^{T}\left(t ; \beta_{j}^{*}\right) \Omega_{j}^{-1}\left(\beta_{j}^{*}\right) n^{-\frac{1}{2}} \sum_{i=1}^{n} U_{i j}\left(\beta_{j}^{*}\right) \\
& +e^{\beta_{j}^{* T} Z_{i}} n^{-\frac{1}{2}} \sum_{i=1}^{n} \int_{0}^{t} \frac{\mathrm{d} M_{i j}^{*}(u)}{r^{(0)}\left(u ; \beta_{j}^{*}\right)}+o_{p}(1),
\end{aligned}
$$

where $K_{i j}\left(t ; \beta_{j}^{*}\right)=\int_{0}^{t}\left\{Z_{i}-\bar{z}_{j}\left(u ; \beta_{j}^{*}\right)\right\} \mathrm{d} \Lambda_{i j}^{*}(u)$.

As for model (3), under the assumed regularity conditions, $\widehat{\theta} \stackrel{p}{\longrightarrow} \theta^{*}$, with $\theta^{*}=\theta$ if model (3) is true, and

$$
n^{\frac{1}{2}}\left(\widehat{\theta}-\theta^{*}\right)=V^{-1}\left(\theta^{*}\right) n^{-\frac{1}{2}} \sum_{i=1}^{n} X_{i}\left\{A_{i}-\operatorname{expit}\left(\theta^{* T} X_{i}\right)\right\}+o_{p}(1)
$$

for $j=0,1$ [33].

Finally, regarding censoring, it is standard result (e.g., [34]) that

$$
n^{\frac{1}{2}}\left\{\widehat{\Lambda}_{j}^{C}(t)-\Lambda_{j}^{C}(t)\right\}=n^{-\frac{1}{2}} \sum_{i=1}^{n} \int_{0}^{t} \frac{\mathrm{d} M_{i j}^{C}(u)}{E\left\{Y_{i j}(u)\right\}}+o_{p}(1),
$$

where $\mathrm{d} M_{i j}^{C}(u)=\mathrm{d} N_{i j}^{C}(u)-Y_{i j}(u) \mathrm{d} \Lambda_{j}^{C}(u)$.

\section{A.2. Consistency}

If model (3) is correct, then $\widehat{\theta} \stackrel{p}{\longrightarrow} \theta$ and $p_{i j}(\widehat{\theta}) \stackrel{p}{\longrightarrow} p_{i j}(\theta)=P\left(A_{i j}=1 \mid Z_{i}\right)$. Considering the denominator of (4), it can be rewritten as $n^{-1} \sum_{i=1}^{n}\left[w_{i j}(\widehat{\theta}) e^{\widehat{\Lambda}_{j}^{C}(u)} Y_{i j}(u)-\left\{w_{i j}(\widehat{\theta})-1\right\} e^{-\widehat{\Lambda}_{i j}(u)}\right]$, and it converges in probability to

$$
\begin{aligned}
& E\left\{\frac{A_{i j}}{p_{i j}(\theta)} e^{\Lambda_{j}^{C}(u)} Y_{i j}(u)\right\}-E\left[\left\{\frac{A_{i j}}{p_{i j}(\theta)}-1\right\} e^{-\Lambda_{i j}^{*}(u)}\right] \\
= & E\left[E\left\{\frac{A_{i j}}{p_{i j}(\theta)} e^{\Lambda_{j}^{C}(u)} Y_{i j}(u) \mid Z_{i}\right\}\right]-E\left\{E\left[\left\{\frac{A_{i j}}{p_{i j}(\theta)}-1\right\} e^{-\Lambda_{i j}^{*}(u)} \mid Z_{i}\right]\right\} \\
= & E\left\{P\left(T_{i}>u \mid A_{i j}=1, Z_{i}\right)\right\} \equiv S^{j}(t) .
\end{aligned}
$$


Similarly, one can obtain that the numerator converges in probability to $-\mathrm{d} S^{j}(u)$ uniformly in $u \in[0, \tau]$. Combining results, we obtain that $\widehat{\Lambda}_{j}(t) \stackrel{p}{\longrightarrow} \Lambda_{j}(t)$ uniformly in $t \in[0, \tau]$. Therefore, by the continuous mapping theorem, $\widehat{S}^{j}(t) \stackrel{p}{\longrightarrow} S^{j}(t)$ uniformly in $t \in[0, \tau]$, and in addition, $\widehat{\mu}_{j}$ and $\widehat{\delta}$ are consistent for $\mu_{j}$ and $\delta$, respectively.

If model (1) is correct, then $\widehat{\beta}_{j} \stackrel{p}{\longrightarrow} \beta_{j}^{*}$ and $\widehat{\Lambda}_{i j}(t) \stackrel{p}{\longrightarrow} \Lambda_{i j}(t)$ uniformly in $t \in[0, \tau]$. The denominator of (4) converges in probability to

$$
\begin{aligned}
& n^{-1} \sum_{i=1}^{n} e^{-\widehat{\Lambda}_{i j}(t)}+n^{-1} \sum_{i=1}^{n} w_{i j}(\widehat{\theta})\left\{e^{\widehat{\Lambda}_{j}^{C}(u)} Y_{i j}(u)-e^{-\widehat{\Lambda}_{i j}(u)}\right\} \\
\stackrel{p}{\longrightarrow} & S^{j}(u)+E\left[w_{i j}\left(\theta^{*}\right)\left\{e^{\Lambda_{j}^{C}(u)} Y_{i j}(u)-e^{-\Lambda_{i j}(u)}\right\}\right] \\
= & S^{j}(u)+E\left\{E\left[w_{i j}\left(\theta^{*}\right)\left\{e^{\Lambda_{j}^{C}(u)} Y_{i j}(u)-e^{-\Lambda_{i j}(u)}\right\} \mid Z_{i}\right]\right\} \\
= & S^{j}(u)+E\left\{\frac{p_{i j}(\theta)}{p_{i j}\left(\theta^{*}\right)} \times 0\right\}=S^{j}(u) .
\end{aligned}
$$

Similarly, the numerator converges in probability to $-\mathrm{d} S^{j}(u)$ uniformly in $u \in[0, \tau]$. Therefore, the proposed estimators for $\Lambda_{i j}(t), \mu_{j}$ and $\delta$ are consistent for the true values when model (1) is correct.

Therefore, the proposed estimators are consistent for the true values when at least one of the working models is correct.

\section{A.3. Asymptotic normality}

In the proofs of asymptotic normality, we do not specify explicitly which working model is correct, and we denote that $\widehat{\beta}_{j} \stackrel{p}{\longrightarrow} \beta_{j}^{*}, \widehat{\Lambda}_{i j}(t) \stackrel{p}{\longrightarrow} \Lambda_{i j}^{*}(t)$, and $\widehat{\theta} \stackrel{p}{\longrightarrow} \theta^{*}$. Let us first consider $n^{\frac{1}{2}}\left\{\widehat{\Lambda}_{j}(t)-\Lambda_{j}(t)\right\}$, which, as we will show, can be approximated by a scaled summation of independent and identically distributed variates. We make the following decomposition:

$$
\begin{aligned}
n^{\frac{1}{2}}\left\{\widehat{\Lambda}_{j}(t)-\Lambda_{j}(t)\right\}= & n^{\frac{1}{2}}\left\{\widehat{\Lambda}_{j}\left(t ; \widehat{\theta}, \widehat{\Lambda}_{i j}, \widehat{\Lambda}_{j}^{C}\right)-\widehat{\Lambda}_{j}\left(t ; \theta^{*}, \widehat{\Lambda}_{i j}, \widehat{\Lambda}_{j}^{C}\right)\right\} \\
& +n^{\frac{1}{2}}\left\{\widehat{\Lambda}_{j}\left(t ; \theta^{*}, \widehat{\Lambda}_{i j}, \widehat{\Lambda}_{j}^{C}\right)-\widehat{\Lambda}_{j}\left(t ; \theta^{*}, \Lambda_{i j}^{*}, \widehat{\Lambda}_{j}^{C}\right)\right\} \\
& +n^{\frac{1}{2}}\left\{\widehat{\Lambda}_{j}\left(t ; \theta^{*}, \Lambda_{i j}^{*}, \widehat{\Lambda}_{j}^{C}\right)-\widehat{\Lambda}_{j}\left(t ; \theta^{*}, \Lambda_{i j}^{*}, \Lambda_{j}^{C}\right)\right\} \\
& +n^{\frac{1}{2}}\left\{\widehat{\Lambda}_{j}\left(t ; \theta^{*}, \Lambda_{i j}^{*}, \Lambda_{j}^{C}\right)-\Lambda_{j}(t)\right\} .
\end{aligned}
$$

By Taylor series expansion and substituting preparation results presented previously, after much algebra, we obtain that

$$
\text { (5) }=B_{j}^{T}\left(t ; \beta_{j}^{*}, \theta^{*}\right) V^{-1}\left(\theta^{*}\right) n^{-\frac{1}{2}} \sum_{i=1}^{n} X_{i}\left\{A_{i j}-p_{i j}\left(\theta^{*}\right)\right\}+o_{p}(1),
$$

where

$$
\begin{aligned}
B_{j}\left(t ; \beta_{j}^{*}, \theta^{*}\right) & =\int_{0}^{t} \frac{E\left[\left\{e^{\Lambda_{j}^{C}(u)} \mathrm{d} N_{i j}(u)-e^{-\Lambda_{i j}^{*}(u)} \mathrm{d} \Lambda_{i j}^{*}(u)\right\} w_{i j}\left(\theta^{*}\right)(-1)\left\{1-p_{i j}\left(\theta^{*}\right)\right\} X_{i}\right]}{D_{j}\left(u ; \beta_{j}^{*}, \theta^{*}\right)} \\
& +\int_{0}^{t}\left\{E\left[\left\{e^{\Lambda_{j}^{C}(u)} Y_{i j}(u)-e^{-\Lambda_{i j}^{*}(u)}\right\} w_{i j}\left(\theta^{*}\right)\left\{1-p_{i j}\left(\theta^{*}\right)\right\} X_{i}\right]\right\} \frac{\mathrm{d} Q_{j}\left(u ; \beta_{j}^{*}, \theta^{*}\right)}{D_{j}^{2}\left(u ; \beta_{j}^{*}, \theta^{*}\right)}, \\
& D_{j}\left(u ; \beta_{j}^{*}, \theta^{*}\right)=E\left[w_{i j}\left(\theta^{*}\right) e^{\Lambda_{j}^{C}(u)} Y_{i j}(u)-\left\{w_{i j}\left(\theta^{*}\right)-1\right\} e^{-\Lambda_{i j}^{*}(u)}\right] \\
& \mathrm{d} Q_{j}\left(u ; \beta_{j}^{*}, \theta^{*}\right)=E\left[w_{i j}\left(\theta^{*}\right) e^{\Lambda_{j}^{C}(u)} \mathrm{d} N_{i j}(u)-\left\{w_{i j}\left(\theta^{*}\right)-1\right\} e^{-\Lambda_{i j}^{*}(u)} \mathrm{d} \Lambda_{i j}^{*}(u)\right],
\end{aligned}
$$


(6) $=F_{j}^{T}\left(t ; \beta_{j}^{*}, \theta^{*}\right) \Omega_{j}^{-1}\left(\beta_{j}^{*}\right) n^{-\frac{1}{2}} \sum_{i=1}^{n} U_{i j}\left(\beta_{j}^{*}\right)+n^{-\frac{1}{2}} \sum_{i=1}^{n} \int_{0}^{t} G_{j}\left(u, t ; \beta_{j}^{*}, \theta^{*}\right) \frac{\mathrm{d} M_{i j}^{*}(u)}{r_{j}^{(0)}\left(u ; \beta_{j}^{*}\right)}+o_{p}(1)$,

where

$$
\begin{aligned}
F_{j}\left(t ; \beta_{j}^{*}, \theta^{*}\right)= & \int_{0}^{t} \frac{E\left[\left\{w_{i j}\left(\theta^{*}\right)-1\right\} e^{-\Lambda_{i j}^{*}(u)} \mathrm{d} \Lambda_{i j}^{*}(u)\left\{K_{i j}\left(u ; \beta_{j}^{*}\right)-Z_{i}+\bar{z}_{j}\left(u ; \beta_{j}^{*}\right)\right\}\right]}{D_{j}\left(u ; \beta_{j}^{*}, \theta^{*}\right)} \\
& -\int_{0}^{t} E\left[\left\{w_{i j}\left(\theta^{*}\right)-1\right\} e^{-\Lambda_{i j}^{*}(u)} K_{i j}\left(u ; \beta_{j}^{*}\right)\right] \frac{\mathrm{d} Q_{j}\left(u ; \beta_{j}^{*}, \theta^{*}\right)}{D_{j}^{2}\left(u ; \beta_{j}^{*}, \theta^{*}\right)}, \\
G_{j}\left(u, t ; \beta_{j}^{*}, \theta^{*}\right)= & \int_{u}^{t} \frac{E\left[\left\{w_{i j}\left(\theta^{*}\right)-1\right\} e^{-\Lambda_{i j}^{*}(s)} e^{\beta_{j}^{* T} Z_{i}} \mathrm{~d} \Lambda_{i j}^{*}(s)\right]}{D_{j}\left(s ; \beta_{j}^{*}, \theta^{*}\right)}-\frac{E\left[\left\{w_{i j}\left(\theta^{*}\right)-1\right\} e^{-\Lambda_{i j}^{*}(u)} e^{\left.\beta_{j}^{* T} Z_{i}\right]}\right.}{D_{j}\left(u ; \beta_{j}^{*}, \theta^{*}\right)} \\
& \left.-\int_{u}^{t} E\left[\left\{w_{i j}\left(\theta^{*}\right)-1\right\} e^{-\Lambda_{i j}^{*}(s)} e^{\beta_{j}^{* T}} Z_{i}\right]\right\} \frac{\mathrm{d} Q_{j}\left(s ; \beta_{j}^{*}, \theta^{*}\right)}{D_{j}^{2}\left(s ; \beta_{j}^{*}, \theta^{*}\right)},
\end{aligned}
$$

and

$$
\text { (7) }=n^{-\frac{1}{2}} \sum_{i=1}^{n} \int_{0}^{t} H_{j}\left(u, t ; \beta_{j}^{*}, \theta^{*}\right) \frac{\mathrm{d} M_{i j}^{C}(u)}{E\left\{Y_{i j}(u)\right\}}+o_{p}(1) \text {, }
$$

where

$H_{j}\left(u, t ; \beta_{j}^{*}, \theta^{*}\right)=\int_{u}^{t} \frac{E\left\{w_{i j}\left(\theta^{*}\right) e^{\Lambda}{ }_{j}^{C}(s)\right.}{D_{j}\left(s ; \beta_{j}^{*}, \theta^{*}\right)}-\int_{u}^{t}\left\{\frac{Q_{j}\left(s ; \beta_{j}^{*}, \theta^{*}\right)}{D_{j}^{2}\left(s ; \beta_{j}^{*}, \theta^{*}\right)} \times E\left[w_{i j}\left(\theta^{*}\right) Y_{i j}(s) e^{\Lambda_{j}^{C}(s)}\right\}\right]$.

Finally, as for the last term, it is straightforward to show that

$$
\text { (8) }=n^{-\frac{1}{2}} \sum_{i=1}^{n} \int_{0}^{t} \frac{w_{i j}\left(\theta^{*}\right) e^{\Lambda_{j}^{C}(u)} \mathrm{d} M_{i j}^{\dagger}(u)-\left\{w_{i j}\left(\theta^{*}\right)-1\right\} e^{-\Lambda_{i j}^{*}(u)}\left\{\mathrm{d} \Lambda_{i j}^{*}(u)-\mathrm{d} \Lambda_{j}(u)\right\}}{D_{j}\left(u ; \beta_{j}^{*}, \theta^{*}\right)}+o_{p}(1),
$$

where $\mathrm{d} M_{i j}^{\dagger}(u)=\mathrm{d} N_{i j}(u)-Y_{i j}(u) \mathrm{d} \Lambda_{j}(u)$.

Combining the aforementioned results, we have shown that we can represent $n^{\frac{1}{2}}\left\{\widehat{\Lambda}_{j}(t)-\Lambda_{j}(t)\right\}$ as $n^{-\frac{1}{2}} \sum_{i=1}^{n} \varphi_{i j}(t)$ plus a term that converges in probability to zero, where

$$
\begin{aligned}
\varphi_{i j}(t)= & B_{j}^{T}\left(t ; \beta_{j}^{*}, \theta^{*}\right) V^{-1}\left(\theta^{*}\right) X_{i}\left\{A_{i j}-p_{i j}\left(\theta^{*}\right)\right\}+F_{j}^{T}\left(t ; \beta_{j}^{*}\right) \Omega_{j}^{-1}\left(\beta_{j}^{*}\right) U_{i j}\left(\beta_{j}^{*}\right) \\
& +\int_{0}^{t} G_{j}\left(u, t ; \beta_{j}^{*}, \theta_{j}\right) \frac{\mathrm{d} M_{i j}^{*}(u)}{r_{j}^{(0)}\left(u ; \beta_{j}\right)}+\int_{0}^{t} H_{j}\left(u, t ; \beta_{j}^{*}, \theta^{*}\right) \frac{\mathrm{d} M_{i j}^{C}(u)}{E\left\{Y_{i j}(u)\right\}} \\
& +\int_{0}^{t} \frac{w_{i j}\left(\theta^{*}\right) e^{\Lambda_{j}^{C}(u)} \mathrm{d} M_{i j}^{\dagger}(u)-\left\{w_{i j}\left(\theta^{*}\right)-1\right\} e^{-\Lambda_{i j}^{*}(u)}\left\{\mathrm{d} \Lambda_{i j}^{*}(u)-\mathrm{d} \Lambda_{j}(u)\right\}}{D_{j}\left(u ; \beta_{j}^{*}, \theta^{*}\right)} .
\end{aligned}
$$

When one of the working models is correct, using similar techniques used in proving consistency of $\widehat{\Lambda}_{i j}(t)$ for $\Lambda_{i j}(t)$, it can be shown that $\varphi_{i j}(t)$ has mean zero and are identically and independently distributed across $i=1, \ldots, n$.

Considering the estimation of $\mu_{j}, n^{\frac{1}{2}}\left(\widehat{\mu}_{j}-\mu_{j}\right)$ can be written as

$$
\begin{aligned}
n^{\frac{1}{2}}\left(\widehat{\mu}_{j}-\mu_{j}\right) & =n^{\frac{1}{2}} \int_{0}^{L} \widehat{S}^{j}(u)-S^{j}(u) \mathrm{d} u \\
& =n^{\frac{1}{2}} \int_{0}^{L} e^{-\widehat{\Lambda}_{j}(u)}-e^{-\Lambda_{j}(u)} \mathrm{d} u \\
& =n^{-\frac{1}{2}} \sum_{i=1}^{n} \phi_{i j}+o_{p}(1),
\end{aligned}
$$


where $\phi_{i j}=-\int_{0}^{L} S^{j}(u) \varphi_{i j}(u) \mathrm{d} u$. When at least one of the two working models is correct, the $\phi_{i j}$ variates are independent and identically distributed with mean 0 . Therefore, $n^{\frac{1}{2}}\left(\widehat{\mu}_{j}-\mu_{j}\right)$ converges to a normal distribution with mean 0 and variance $E\left(\phi_{i j}^{2}\right)$. It then follows that $n^{\frac{1}{2}}(\widehat{\delta}-\delta)$ is also asymptotically normal with mean 0 and variance $E\left(\phi_{i 1}-\phi_{i 0}\right)^{2}$ and $n^{\frac{1}{2}}(\widehat{\delta}-\delta)=n^{-\frac{1}{2}} \sum_{i=1}^{n}\left(\phi_{i 1}-\phi_{i 0}\right)+o_{p}(1)$.

\section{Acknowledgements}

This work was supported in part by the National Institutes of Health grant 5R01-DK070869. The authors thank Maastricht University Medical Center, for the access to the kidney transplant data. They also thank three anonymous referees whose comments strengthened the manuscript considerably.

\section{References}

1. Kaplan EL, Meier P. Nonparametric estimation from incomplete observations. Journal of American Statistical Association 1958; 53:457-481. DOI: 10.1080/01621459.1958.10501452.

2. Cox DR. Regression models and life tables (with Discussion). Journal of the Royal Statistical Society, Series B 1972; 34:187-200.

3. Karrison T. Restricted mean life with adjustment for covariates. Journal of the American Statistical Association 1987; 82:1169-1176. DOI: 10.1080/01621459.1987.10478555.

4. Karrison TG. Use of Irwin's restricted mean as an index for comparing survival in different treatment groups-Interpretation and power considerations. Controlled Clinical Trials 1997; 18:151-167. DOI: 10.1016/S0197-2456(96)00089-X.

5. Zucker DM. Restricted mean life with covariates: modification and extension of a useful survival analysis method. Journal of the American Statistical Association 1998; 93:702-709. DOI: 10.1080/01621459.1998.10473722.

6. Andersen PK, Hansen MG, Klein JP. Regression analysis of restricted mean survival time based on pseudo-observations. Lifetime Data Analysis 2004; 10:335-350. DOI: 10.1007/s10985-004-4771-0.

7. Schaubel DE, Guidinger MK, Biggins SW, Kalbfleisch JD, Pomfret EA, Sharma P, Merion RM. Survival benefit-based deceased-donor liver allocation. American Journal of Transplantation 2009; 9(4 Pt 2):970-981. DOI: 10.1111/j.16006143.2009.02571.x.

8. Meier P, Karrison T, Chappell R, Xie H. The price of Kaplan-Meier. Journal of the American Statistical Association 2004; 99:890-896. DOI: 10.1198/016214504000001259.

9. Andersen PK, Perme MK. Pseudo-observations in survival analysis. Statistical Methods in Medical Research 2010; 19:71-99. DOI: 10.1177/0962280209105020.

10. Chen P, Tsiatis AA. Causal inference on the difference of the restricted mean life between two groups. Biometrics 2001; 57:1030-1038. DOI: 10.1111/j.0006-341X.2001.01030.x.

11. Rotnitzky A, Robins JM. Inverse probability weighting in survival analysis. The Encyclopedia of Biostatistics 2005; 4:2619-2625. DOI: 10.1002/0470011815.b2a11040.

12. Robins JM, Hernán M, Brumback B. Marginal structural models and causal inference in epidemiology. Epidemiology 2000; 11:550-560. DOI: 10.1097/00001648-200009000-00011.

13. Xie J, Liu C. Adjusted Kaplan-Meier estimator and log-rank test with inverse probability of treatment weighting for survival data. Statistics in Medicine 2005; 24:3089-3110. DOI: 10.1002/sim.2174.

14. Cole SR, Hernàn MA. Adjusted survival curves with inverse probability weights. Computer Methods and Programs in Biomedicine 2004; 75:45-49. DOI: 10.1016/j.cmpb.2003.10.004.

15. Wei G. Semiparametric methods for estimating cumulative treatment effects in the presence of non-proportional hazards and dependent censoring. Doctoral Dissertation, Department of Biostatistics, university of Michigan, 2008.

16. Hubbard A, van der Laan MJ, Robins JM. Nonparametric locally efficient estimation of the treatment specific survival distribution with right censored data and covariates in observational studies. Statistical Models in Epidemiology, the Environment and Clinical trials; Springer Verlag 1999; 116:135-178. DOI: 10.1007/978-1-4612-1284-3_3.

17. Rosenbaum PR, Rubin DB. The central role of the propensity score in observational studies for causal effects. Biometrika 1983; 70:41-55. DOI: 10.1093/biomet/70.1.41.

18. Ho DE, Imai K, King G, Stuart EA. Matching as nonparametric preprocessing for reducing model dependence in parametric causal inference. Political science 2007; 15:199-236. DOI: 10.1093/pan/mpl013.

19. Bang H, Robins JM. Doubly robust estimation in missing data and causal inference models. Biometrics 2005; 61:962-972. DOI: $10.1111 /$ j.1541-0420.2005.00377.x.

20. Kang JDY, Schafer JL. Demystifying double robustness: a comparison of alternative strategies for estimating population means from incomplete data. Statistical Science 2007; 26:523-539. DOI: 10.1214/07-STS227.

21. Robins JM, Rotnitzky A, Zhao LP. Estimation of regression coefficients when some regressors are not always observed. Journal of the American Statistical Association 1994; 89:846-866. DOI: 10.1080/01621459.1994.10476818.

22. Snoeijs MG, Schaubal DE, Hene R, Hoitsma AJ, Idu MM, IJzermans JN, Ploeg RJ, Ringers J, Christiaans MH, Buurman WA, van Heurn LWE. Kidneys from donors after cardiac death provide survival benefit. Journal of the American Society of Nephrology 2010; 21:1015-1021. DOI: 10.1681/ASN.2009121203.

23. Rubin DB. Discussion of "Randomization Analysis of Experimental Data in the Fisher Randomization Test," by D. Basu. Journal of the American Statistical Association 1980; 75:591-593. DOI: 10.2307/2287653. 


\section{Statistics}

24. Rubin DB. Which ifs have causal answers? Discussion of Holland's "Statistics and causal inference." Journal of the American Statistical Association 1986; 81:961-962. DOI: 10.2307/2289065.

25. Heckman JJ, Ichimura H, Todd PE. Matching as an econometric evaluation estimator: evidence from evaluating a job training programme. The Review of Economic Studies 1997; 64:605-654. DOI: 10.2307/2971733.

26. Heckman JJ. The scientific model of causality. Sociological Methodology 2005; 35:1-97. DOI: 10.1111/j.0081-1750. 2005.00164.x.

27. Cox DR. Partial likelihood. Biometrika 1975; 62:269-275. DOI: 10.2307/2335362.

28. Breslow NE. Contribution to the discussion on the paper by D. R. Cox, regression and life tables. Journal of the Royal Statistical Society, Series B 1972; 34:216-217.

29. Tsiatis AA. Semiparametric Theory and Missing Data. Springer: New York, 2006.

30. Schaubel DE, Wei G. Double inverse weighted estimation of cumulative treatment effects under non-proportional hazards and dependent censoring. Biometrics 2011; 67:29-38. DOI: 10.1111/j.1541-0420.2010.01449.x.

31. Hirano K, Imbens GW. Estimation of causal effects using propensity score weighting: an application to data on right hear catheterization. Health Services \& Outcomes Research Methodology 2001; 2:259-278. DOI: 10.1023/A:1020371312283.

32. Lin DY, Wei LJ. The robust inference for the Cox proportional hazards model. Journal of the American Statistical Association 1989; 84:1074-1078. DOI: 10.1080/01621459.1989.10478874.

33. Zeng D, Chen Q. Adjustment for missingness using auxiliary information in semiparametric regression. Biometrics 2009; 66:115-122. DOI: 10.1111/j.1541-0420.2009.01231.x.

34. Fleming TR, Harrington DP. Counting Processes and Survival Analysis. John Wiley and Sons: New York, 1991. 